$12-31-2012$

\title{
Separation Control on High Lift Low-Pressure Turbine Airfoils Using Pulsed Vortex Generator Jets
}

\author{
Ralph J. Volino \\ U.S. Naval Academy
}

Mounir B. Ibrahim

Cleveland State University, m.ibrahim@csuohio.edu

Follow this and additional works at: https://engagedscholarship.csuohio.edu/enme_facpub

Part of the Mechanical Engineering Commons

How does access to this work benefit you? Let us know!

\section{Original Citation}

Volino, R. J., and Ibrahim, M. B., 2012, "Separation Control on High Lift Low-Pressure Turbine Airfoils using Pulsed Vortex Generator Jets," Applied Thermal Engineering, 49(0) pp. 31-40.

This Article is brought to you for free and open access by the Mechanical Engineering Department at EngagedScholarship@CSU. It has been accepted for inclusion in Mechanical Engineering Faculty Publications by an authorized administrator of EngagedScholarship@CSU. For more information, please contact library.es@csuohio.edu. 


\title{
Separation control on high lift low-pressure turbine airfoils using pulsed vortex generator jets
}

\author{
Ralph J. Volino ${ }^{\mathrm{a}, *}$, Mounir B. Ibrahim ${ }^{\mathrm{b}}$ \\ a United States Naval Academy, Mechanical Engineering Department, 590 Holloway Road, Annapolis, MD 21402, USA \\ ${ }^{\mathrm{b}}$ Cleveland State University, Mechanical Engineering Department, Stilwell Hall, Room 261, 1960 East 24th Street, Cleveland, OH 44115, USA
}

\section{Introduction}

Gas turbine engines power nearly all commercial and military aircraft and are used extensively for ship propulsion and land based power generation. Given the hundreds of billions of liters of fuel used in gas turbines each year, any improvement in their efficiency, even an improvement of only $1 \%$, would result in a huge savings in fuel and reduction in emissions. The low-pressure turbine (LPT) is the heaviest component in a gas turbine engine and produces the net power from the engine. Increases in LPT component efficiency result in almost equal increase in overall engine efficiency [1]. One way to potentially improve the LPT is to reduce part count, weight and cost through the use of very highly loaded blading. The limitation on loading is boundary layer separation, which leads to partial loss of lift and higher aerodynamic losses (e.g. Mayle [2]).

Separation and separated flow transition, which can lead to boundary layer reattachment, have received considerable attention, as noted by Volino [3,4]. In general, previous work shows that

\footnotetext{
* Corresponding author. Tel.: +1 410293 6520; fax: +1 4102933041.

E-mail addresses: volino@usna.edu (R.J. Volino), m.ibrahim@csuohio.edu (M.B. Ibrahim).
}

the strong acceleration on the leading section of the airfoil keeps the boundary layer thin and laminar. When separation does occur, it is usually just downstream of the suction peak. If transition then occurs in the shear layer over the separation bubble, it is typically rapid and often causes the boundary layer to reattach $[4,5]$.

Airfoils can be designed with high resistance to separation, as described by Praisner and Clark [6], but a loading limit will always exist, above which separation will still occur. If flow control were incorporated in the design of an advanced airfoil, it might be possible to increase the loading limit. Passive devices such as boundary layer trips (eg. Zhang et al. [7] and Bohl and Volino [8]) have been shown effective for separation control and have the distinct advantage of simplicity, but they also introduce parasitic losses and cannot be adjusted to account for changes in flow conditions. Active devices would be more costly and potentially risky in terms of reliability, but could potentially provide better control over the entire operating range of interest.

The literature contains many examples of active separation control. In turbomachinery, plasma devices, as used by Huang et al. [9], could be viable. Vortex generator jets (VGJs), as introduced by Johnston and Nishi [10], are another alternative and the subject of the present study. Blowing from small, compound angled holes is 
used to create streamwise vortices. The vortices bring high momentum fluid into the near wall region, which can help to control separation. The most effective VGJs enter the boundary layer at a relatively shallow pitch angle (typically $30-45^{\circ}$ ) relative to the wall and a high skew angle $\left(45-90^{\circ}\right)$ relative to the main flow. The jets also promote transition, and turbulent mixing helps to mitigate separation. Several studies (e.g [11,12].) used VGJs on the highly loaded Pack B LPT airfoil. Separation was essentially eliminated, even at the lowest Reynolds number considered ( $R e=25000$ based on suction surface length and nominal exit velocity). Pulsed jets were more effective than steady jets. The initial disturbance created by each pulse caused the boundary layer to attach. The turbulence was followed by a calmed period (Gostelow et al. [13] and Schulte and Hodson [14]) during which the boundary layer was very resistant to separation but very laminarlike in terms of its fluctuation levels and low losses. When the time between pulses was long enough, the boundary layer did eventually relax to a separated state, but due to the control which persisted during the calmed period, the VGJs were effective even with low jet pulsing frequencies, duty cycles and mass flow rates. Since the boundary layer was attached and undisturbed for much of the jet pulsing cycle, profile losses were low.

In the present study, the very highly loaded L1A airfoil is used. The L1A is an aft loaded blade designed at the Air Force Research Laboratory (AFRL) and available on a limited basis from Clark [15]. Dimensions as used in the present study are given in Table 1 . The L1A has a Zweifel coefficient of 1.35 , which corresponds to $10 \%$ higher loading than the "ultra-high lift" airfoils described by Zhang and Hodson [7], and 17\% higher loading than the Pack B. Because the L1A is highly loaded and aft loaded, it is prone to separation, as documented in Bons et al. [16] and Volino [3]. In cases without flow control and low Reynolds numbers, the boundary layer separates and does not reattach, in spite of transition to turbulence in the shear layer over the separation bubble. This result contrasts with the results of studies on less aggressive airfoils, which all showed reattachment after transition. The failure to reattach results in a 20\% loss in lift and increases profile losses by up to a factor of 7 compared to high Reynolds number cases.

Separation control with VGJs has been demonstrated on the L1A airfoil by Bons et al. [16], who considered a case with $R e=50000$, background freestream turbulence $T I=3 \%$, and periodic wakes, and by Volino et al. [17], who considered cases with $T I=0.6 \%$ and documented pressure distributions on the airfoils and total pressure losses. Cases were considered at Reynolds numbers from 25000 to 100000 (10000-40000 based on inlet velocity and axial chord). Jet pulsing frequency was varied from $F=0.14$ to 1.12 with duty cycles of $10 \%$ and $50 \%$ and blowing ratios (defined as the ratio of maximum velocity in the pulse to the local freestream velocity) ranging from 0.25 to 3.0 . In agreement with previous studies, pulsed jets were more effective than steady jets. Separation control was achieved at $R e=25000$ with $B=0.75, F=0.56$ and $D=10 \%$, and at $\operatorname{Re}=50000$ with $F=0.56, B=0.25$ and $D=10 \%$. Partial control was possible at lower frequencies, particularly with higher blowing ratios or duty cycles. Lower frequencies were more effective at $R e=50000$ than at $R e=25000$. Effective separation control resulted in a $20 \%$ increase in lift, and up to a $60 \%$ reduction in total

Table 1

Cascade parameters.

\begin{tabular}{lllllll}
\hline $\begin{array}{l}\text { Axial } \\
\text { Chord, } \\
C_{x}[\mathrm{~mm}]\end{array}$ & $\begin{array}{l}\text { True } \\
\text { Chord }\end{array}$ & $\begin{array}{l}\text { Pitch, } \\
{[\mathrm{mm}]}\end{array}$ & $\begin{array}{l}\text { Span } \\
{[\mathrm{mm}]}\end{array}$ & $\begin{array}{l}\text { Suction } \\
{[\mathrm{mm}]}\end{array}$ & $\begin{array}{l}\text { Inlet } \\
\text { side, } \\
L_{s}[\mathrm{~mm}]\end{array}$ & $\begin{array}{l}\text { Exit } \\
\text { angle }\end{array}$ \\
\hline 134 & 146 & 136 & 724 & 203 & $\begin{array}{l}\text { flow } \\
\text { angle }\end{array}$ \\
\hline
\end{tabular}

pressure loss, dropping from about 5 times the high $R e$ value to about twice the high $R e$ value.

In the present study, the cases of Volino et al. [17] are examined in more detail. In addition to the measurements presented previously, velocity in the suction surface boundary layer is used to document and explain the flow separation and reattachment, and the effect of the VGJs.

\section{Experimental facility and measurements}

Experiments were conducted in a closed loop wind tunnel with a seven blade linear cascade located in the wind tunnel's third turn, as shown in Fig. 1a. A fine screen located upstream of the cascade is
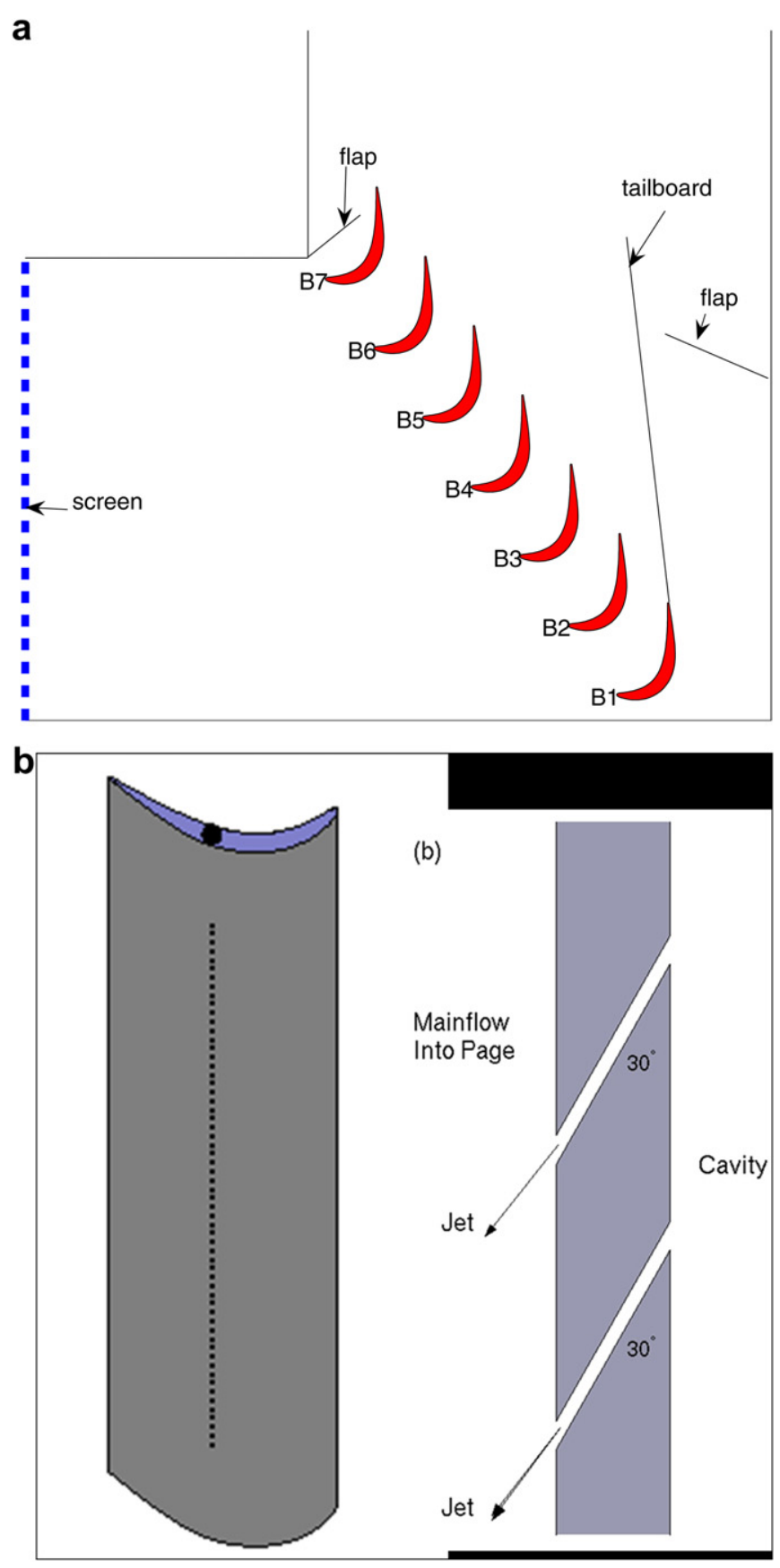

Fig. 1. Drawings of test section: a) linear cascade, b) airfoil with VGJ holes and cross section of hole geometry. 

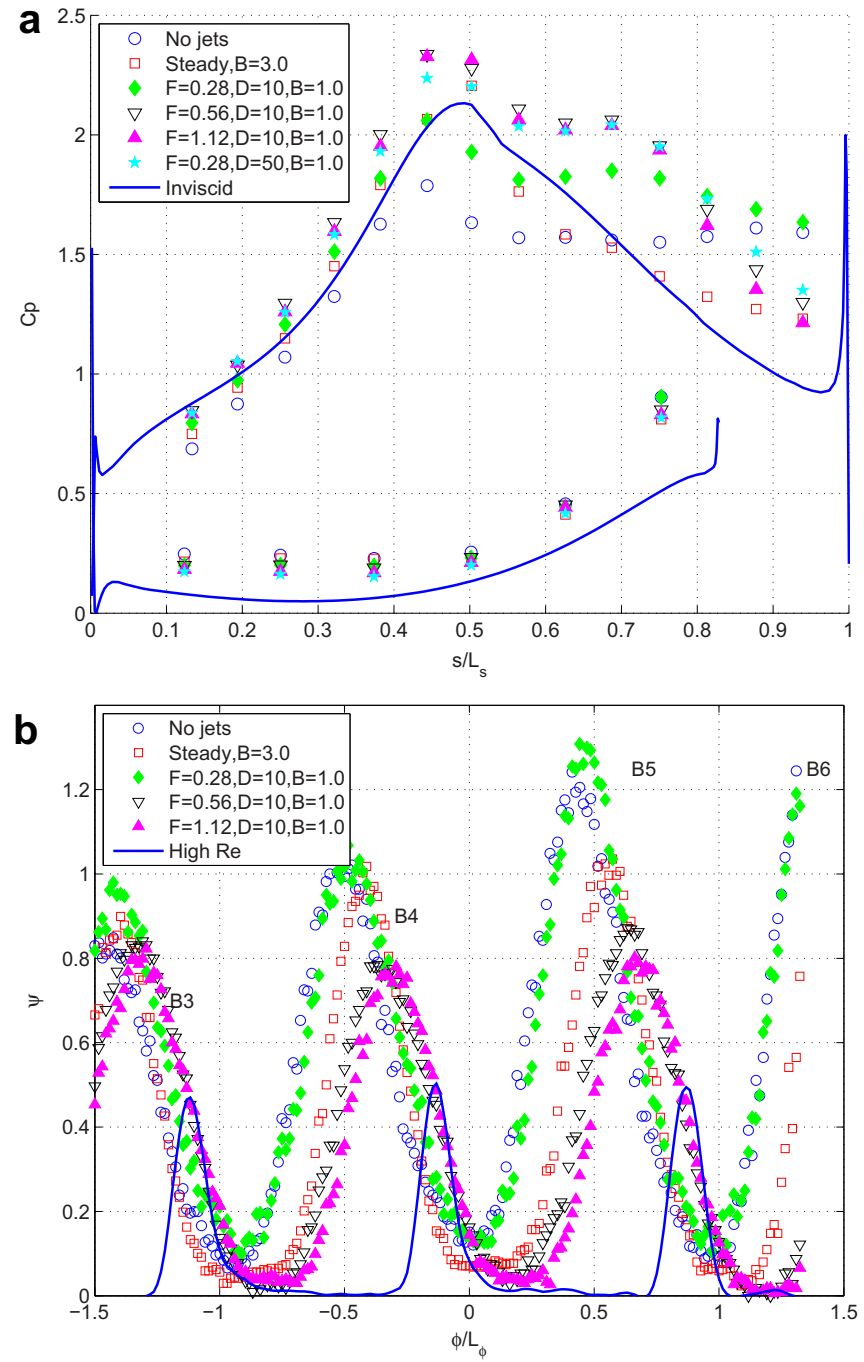

Fig. 2. Pressure results for $R e=25000$ cases: a) $\mathrm{Cp}$, b) total pressure loss.

used to provide uniform inlet conditions and $0.6 \%$ freestream turbulence intensity as noted in Volino [3]. The freestream turbulence intensity in an engine is expected to be of the order $4 \%$, which would result in more rapid transition of the boundary layer and resistance to separation, as shown in Volino et al. [18]. The effect of wakes from upstream airfoils would be similar. The present experiments provide a more challenging case for flow control and a baseline for cases with higher freestream turbulence and wakes, some of which are documented in Volino et al. [19]. In Volino et al. [19] it is shown the freestream turbulence has a small effect compared to the VGJ flow control.

A tailboard, shown in Fig. 1a, was needed to produce the correct exit flow angle from the cascade. Its position was set to produce periodicity at high Reynolds numbers. A tailboard on the opposite side of the cascade, and inlet guide vanes were found to be unnecessary. To produce the correct approach flow to the end blades (B1 and B7), the amount of flow escaping around the two ends of the cascade was controlled with the flaps shown in Fig. 1a. The flap positions were set using a wool tuft upstream of each blade to check that the incoming flow approached the stagnation points with the correct angle. The inlet flow angle was also checked with a three-hole pressure probe and found to be within $2^{\circ}$ of the design angle. At high Reynolds numbers, the approach velocity to the middle four passages was measured to be uniform to within $6 \%$, and the difference between any two adjacent passages was within 3\%. At low Reynolds numbers, slightly more variation was observed, but the approach velocity to the middle two passages still agreed to within 5\%. Good periodicity at high Reynolds numbers was also observed in the exit flow from the cascade, as evidenced by suction side velocity profiles acquired near the trailing edge of blades B2$\mathrm{B} 6$, and by total pressure loss surveys, which are shown below. At low Reynolds numbers, when significant separation bubbles were present, the periodicity was not as good due to suppression of the separation bubble thickness on the blades closest to the tailboard. This is an unavoidable result when using a finite linear cascade to study separated flow. It is considered acceptable for the present facility, since its intended purpose is for the study of flow control, which when successful suppresses separation on all blades, thereby restoring periodicity even at low Reynolds numbers.

Each blade in the cascade has a central cavity which extends along the entire span. As explained in Volino et al. [17], compressed air is supplied to the cavities through fast response solenoid valves. A single spanwise row of holes was drilled into the suction surface of each blade at the inviscid pressure minimum location, $s / L_{s}=0.5$ $\left(x / C_{x}=0.62\right)$, which is about the optimal location for flow control devices. The holes are $0.8 \mathrm{~mm}$ in diameter and drilled at $30^{\circ}$ to the surface and $90^{\circ}$ to the main flow direction, as shown in Fig. $1 \mathrm{~b}$. The hole spacing is 10.6 diameters, and the length to diameter ratio is
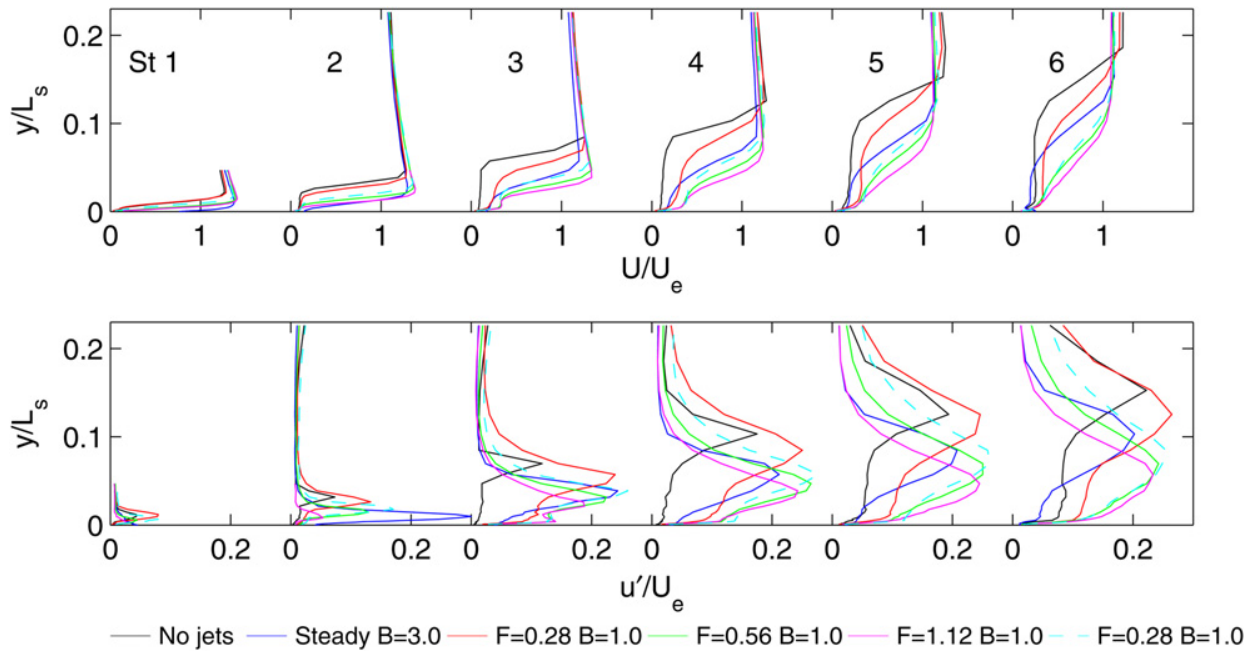

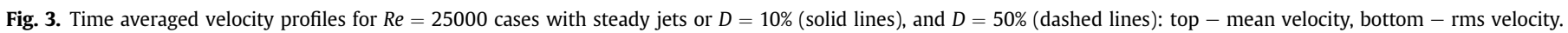


Table 2

Velocity profile measurement stations.

\begin{tabular}{lllllll}
\hline Station & 1 & 2 & 3 & 4 & 5 & 6 \\
\hline$s / L_{s}$ & 0.53 & 0.59 & 0.69 & 0.78 & 0.88 & 0.97 \\
$x / C_{x}$ & 0.65 & 0.72 & 0.80 & 0.86 & 0.92 & 0.97 \\
\hline
\end{tabular}

12. With steady blowing and $B=1$, the mass flow rate of the jets is $0.04 \%$ of the main flow. With pulsed jets the mass flow is proportionally lower. The solenoid valves pulse the VGJs, and the pulsing frequency in dimensionless form is $F=f L_{j-t e} / U_{\text {ave }}$. Blowing ratio for pulsed jets is the ratio of maximum velocity in the pulse to local freestream velocity, as explained in Volino et al. [17].

The center blade, designated B4 in Fig. 1, contains pressure taps near the spanwise centerline. Stagnation pressure is measured with a pitot tube upstream of the cascade. The uncertainty in the suction side pressure coefficients, $C p$, is 0.07 . Total pressure losses are documented using a Kiel probe traversed across three blade spacings, $0.63 C_{x}$ downstream of the cascade.

Velocity profiles in the suction surface boundary layer were measured near the midspan at the six streamwise stations listed in Table 2 with a hot-wire probe. At each measurement location, data were acquired for $26 \mathrm{~s}$ at a $20 \mathrm{kHz}$ sampling rate ( $2^{19}$ samples). Data were acquired at 40 wall normal locations in each profile. The data were both time averaged and ensemble averaged based on the phase within the jet pulsing cycle at 24 dimensionless times, $t / T$, within the pulsing cycle. Flow direction in a separation bubble cannot be determined with a single-sensor hot-wire, but velocity magnitude can be measured and was found to be near zero within the bubbles of the present cases when the flow was laminar. In cases where the flow became turbulent but remained separated, fluctuating velocities caused false high mean velocity readings in the separation bubble. With the exception of these turbulent separated cases, the uncertainty in the mean velocity is $3-5 \%$

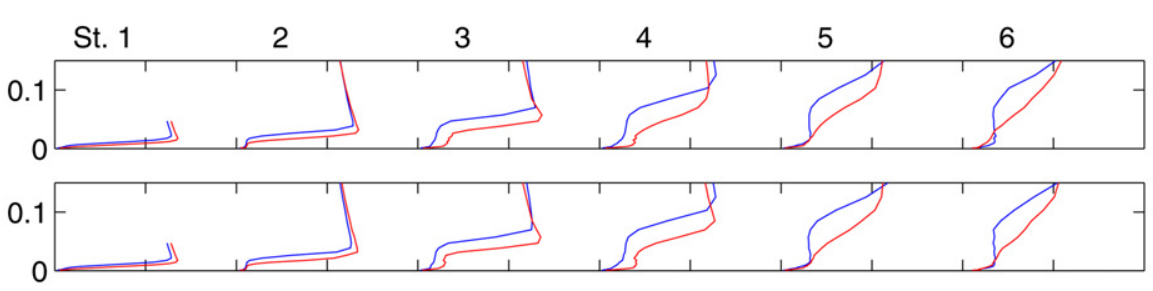

$\mathrm{t} / \mathrm{T}$

0.083
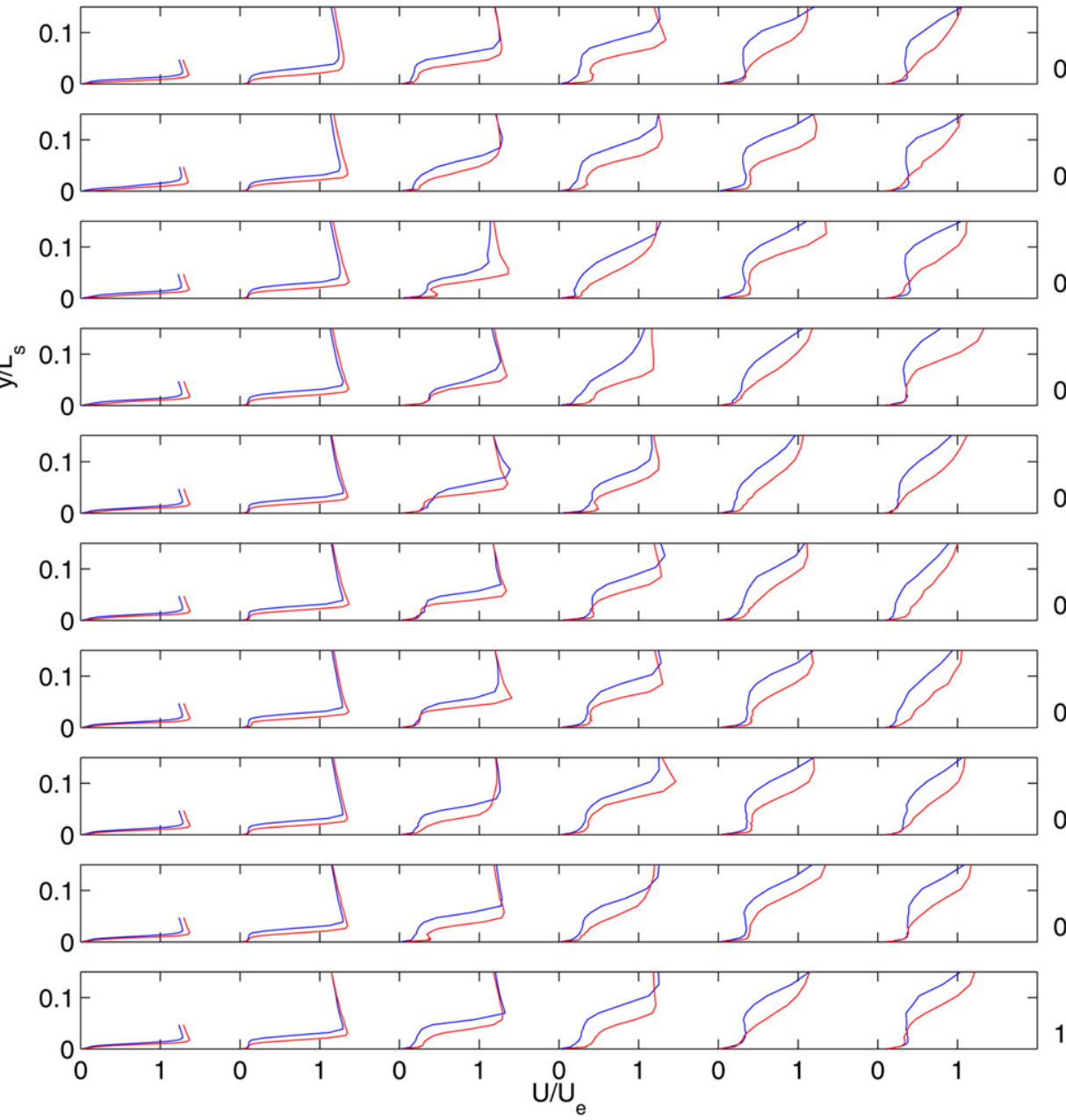

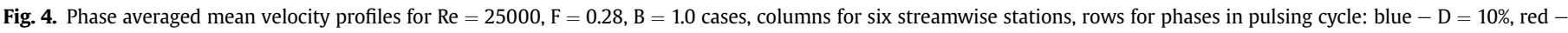
$\mathrm{D}=50 \%$. (For interpretation of the references to colour in this figure legend, the reader is referred to the web version of this article.) 

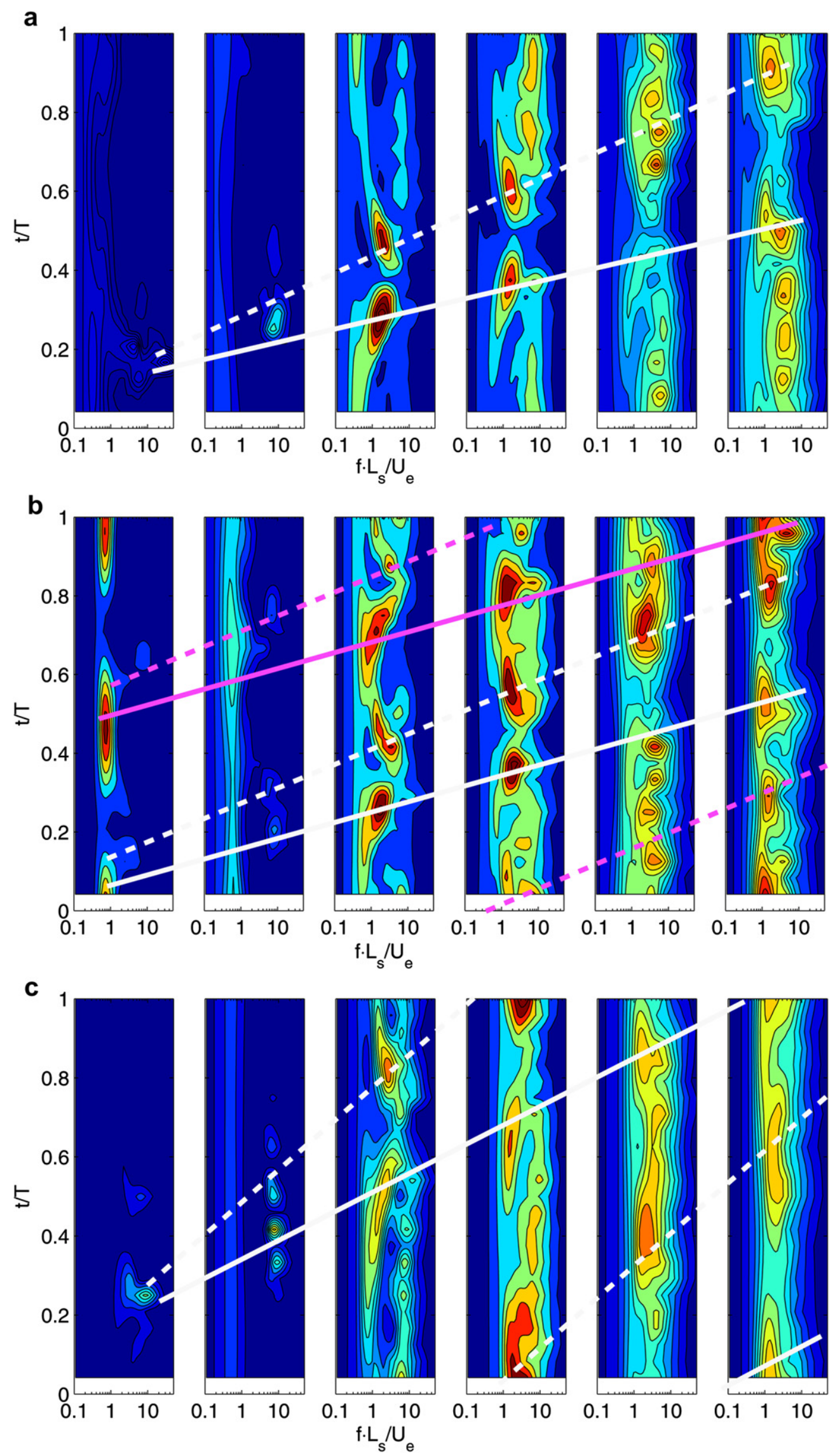

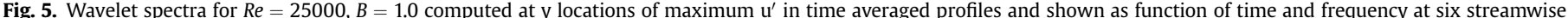
stations: a) $F=0.28, D=10 \%$, b) $F=0.28, D=50 \%$, c) $F=0.56, D=10 \%$. 
except in the very near wall region, where near-wall corrections (Wills [20]) were applied to the mean velocity. The uncertainty in the fluctuating streamwise velocity is below $10 \%$.

Wavelet spectra of the fluctuating velocity were computed using the method described in Volino [21]. In contrast to Fourier spectra, in which a signal is transformed from the time domain to the frequency domain, wavelet spectra provide the frequency content of a signal on a time resolved basis. Wavelet spectra are computed as the convolution integral of a signal (the fluctuating velocity) and a function containing oscillations of a frequency of interest. The function is known as the wavelet. The result of the convolution indicates the times when the frequency of interest is present in the original signal and the magnitude of these fluctuations. By dilating or compressing the wavelet along the time axis, it's frequency can be changed, and the convolution integral with the signal is repeated to determine the presence of other frequencies of interest. After several frequencies have been considered, a wavelet map can be constructed, as shown in the figures below. The Mexican Hat wavelet was used for the present analysis. The wavelet spectra were ensemble averaged to show frequency as a function of phase within the pulsing cycle.

\section{Results}

\section{1. $R e=25000$}

Pressure profiles for cases with $R e=25000$ are shown in Fig. 2 . The inviscid profile for the L1A airfoil is shown as a reference. The low peak followed by a plateau in the case without jets indicates separation without reattachment. As shown in Volino et al. [17], steady blowing with $B=2.0$ or lower has no effect on separation, but with $B=3.0$, there are some signs of reattachment, although the $C p$ profile remains significantly different from the inviscid profile. With $B=1.0$, pulsed jets with $F=0.28$ and $D=10 \%$ have little effect, but with $F=0.28$ and $D=50 \%$ the $C p$ values drop near the trailing edge, indicating some reattachment after a large separation bubble. The $F=0.56$ and $F=1.12$ profiles with $D=10 \%$ are very similar to the $F=0.28, D=50 \%$ case. Total pressure loss profiles are shown in Fig. $2 \mathrm{~b}$ and agree with the $C p$ profiles. As the $C p$ profiles show more reattachment and a smaller separation bubble, the loss profiles show lower loss peaks and a shift to the right, which indicates more flow turning due to a smaller separation bubble. A high Reynolds number (300000) case from Volino [3] is shown for comparison. The loss, $\psi$, is shown as a function of distance across the cascade, $\varphi$, normalized on the blade spacing $\mathrm{L}_{\varphi}$. The origin, $\varphi=0$, corresponds to the location downstream of the trailing edge of the center blade (B4 in Fig. 1a) in the flow direction. Steady blowing with $B=3.0$ reduces the loss peaks somewhat, reestablishes periodicity, and causes the loss peaks to shift to the right. The shift indicates an increase in flow turning of about $6^{\circ}$. The integrated total pressure loss for the passage, however, actually increases by $28 \%$ over the no-jet case due to the losses in the jets between the plenum and hole exit, as documented in Volino et al. [17]. Pulsed jets at $F=0.28, D=10 \%$ and $B=1.0$ have no effect in reducing losses. The $F=0.56$ and 1.12 cases with $D=10 \%$ are very similar to each other and have about $29 \%$ lower losses than the nojet case. The flow turning is increased by about $9^{\circ}$ compared to the case without jets. In these higher $F$ cases the integrated loss is still almost 4 times higher and the flow turning is $4^{\circ}$ lower than the high $R e$ case. The exit flow angle for the high Re case is within $1^{\circ}$ of the design exit flow angle.

Fig. 3 shows time averaged velocity profiles. The top row shows the mean velocity at the six streamwise stations of Table 2 , and the lower row shows the rms fluctuating streamwise velocity, $u^{\prime}$. Without flow control, the boundary layer has separated by Station 1 and the separation bubble grows at the downstream stations. The peak in $u^{\prime}$ is in the shear layer far from the wall. With steady $B=3.0$, $u^{\prime}$ is higher at the upstream stations, and separation appears to be delayed until Station 2 . The boundary layer still separates, however and does not reattach. The separation bubble is about $3 / 4$ the thickness of the no-jet case. The reduction in bubble thickness agrees with the slightly lower loss peaks and somewhat greater flow turning in Fig. 2 compared to the no-jet case. With $F=0.28$ and $D=10 \%, u^{\prime}$ is higher and the shear layer slightly thicker than in the no-jet case, but there is no significant change in the separation bubble thickness, in agreement with the Fig. 2 results. With $F=0.28$ and $D=50 \%$ and with $F=0.56$ or 1.12 and $D=10 \%$, a separation bubble is still visible between Stations 2 and 5 , but it is much thinner than in the other cases, and the boundary layer is reattached by the trailing edge. The peak in $u^{\prime}$ is close to the wall.

Phase averaged mean velocity profiles for the $F=0.28$ cases are shown in Fig. 4. The columns correspond to the six streamwise stations, and rows are for different phases in the pulsing cycle. With $D=10 \%$, the boundary layer separates and does not reattach at most phases. The low, nearly constant velocity near the wall indicates the bubble. The measured velocity is not zero in the bubble because the hot-wire cannot distinguish direction, so reversed flow and turbulence cause a false positive mean velocity in the bubble. At $t / T=0.333$,
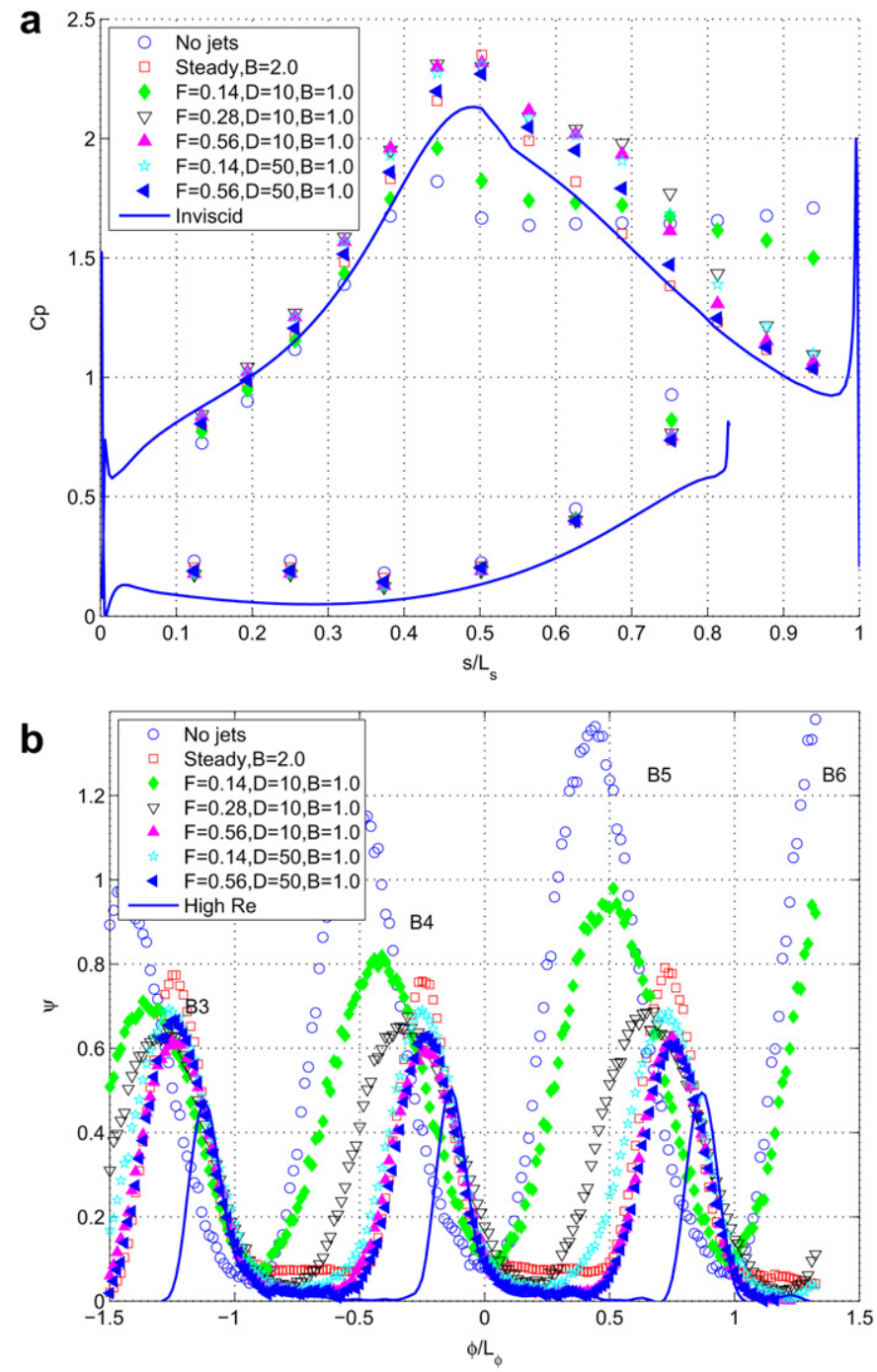

Fig. 6. Pressure results for $R e=50000$ cases: a) Cp, b) total pressure loss. 

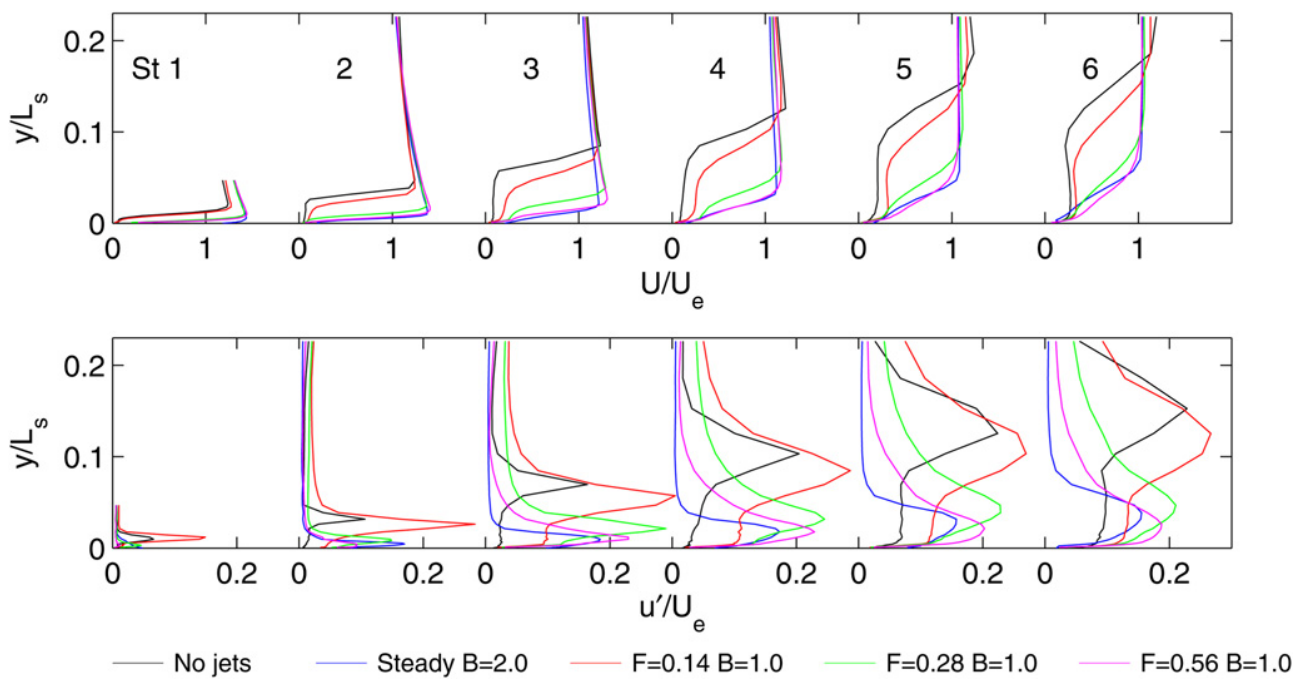

Fig. 7. Time averaged velocity profiles at six streamwise stations for $R e=50000$ cases with steady jets or $D=10 \%$ : top - mean velocity, bottom - rms velocity.

$0.5,0.583$, and 0.667 to 0.75 at Stations $3-6$ respectively, the near wall region of low velocity is less apparent and the velocity goes more continuously toward zero at the wall. This indicates reattachment at these phases. Fig. 5a shows wavelet spectra for the $F=0.28, D=10 \%$ case. The six plots in the figure correspond to the six streamwise stations. In each plot, the horizontal axis shows dimensionless frequency, $f L_{s} / U_{e}$ on a log scale, and the vertical axis shows dimensionless time, $t / T$, for one pulsing cycle. Power spectral density is computed from instantaneous velocity data at all $y$ locations and is shown for the $y$ location corresponding to maximum time averaged $u^{\prime}$ at each station. The contours show the power spectral density premultiplied by frequency and normalized by $U_{e}^{2}$. The color scale is the same for all plots. The VGJs creates a disturbance at the beginning of a pulse, and the leading edge of this disturbance is visible as high contours centered at $t / T=0.11,0.24,0.26,0.36,0.43$ and 0.49 at Stations $1-6$ respectively. The arrival times at each station indicate that the leading edge of the disturbance convects along the surface at about 0.5 times the local freestream velocity (marked by solid white line). A second peak appears centered at $t / T=0.19,0.39,0.46,0.60$, 0.75 and 0.91 at Stations $1-6$ respectively. These peaks are believed to result when the trailing edge of the VGJ disturbance passes, and the times indicate a convection speed of about 0.3 times the freestream velocity (marked by dashed white line). The leading and trailing edge convection speeds are typical of disturbances in boundary layers, as documented by others such as Stieger and Hodson [22] and Zhang and Hodson [23]. The arrival times of the disturbance in Fig. 5 are just prior to the reattachment times in Fig. 4. With the strong adverse pressure gradient, the boundary layer separates again even before the trailing edge of the disturbance passes. Peaks appear in Fig. 5 at the dimensionless frequency, $f L_{s} / U_{e}=1.4$, and tails of these peaks extend to the pulsing frequency, which is 0.7 for this case. A higher frequency peak at $f L_{S} / U_{e} \approx 7$ is also visible, particularly at Stations 1 and 2 . The higher frequency is likely related to shear layer transition, and matches the frequency peak observed in transition without flow control in Volino et al. [3]. At the downstream stations there is a broader range of frequencies for the full cycle, since the shear layer has transitioned to turbulent.

When the duty cycle is increased to $50 \%$ with $F=0.28$, the separation bubble is thinner. Fig. 4 shows boundary layer reattachment at about the same phases as in the $D=10 \%$ case. It then
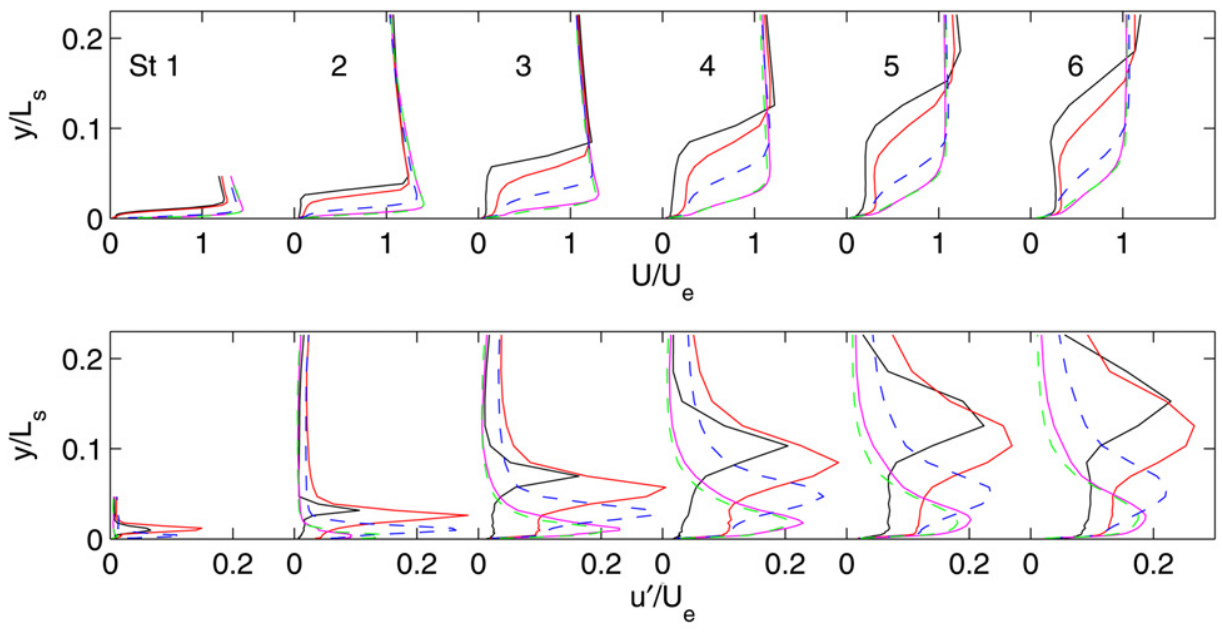

No jets

$F=0.14 D=10 \%$

$F=0.56 D=10 \%$

$F=0.14 D=50 \%$

$F=0.56 D=50 \%$

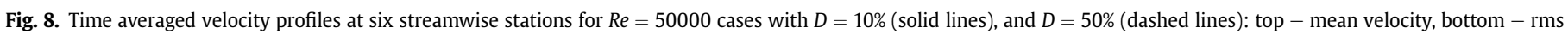
velocity. 
starts to re-separate, but at Stations 5 and 6 it reattaches again about half a cycle later. This second reattachment means less time for the separation bubble to grow, resulting in a thinner separation at all times. The wavelet spectra of Fig. 5b show the disturbance responsible for the second reattachment. When the VGJ turns on, it creates a disturbance much like that in the $D=10 \%$ case. When the jet turns off half a cycle later, it creates a second disturbance which moves down the surface at about the same velocity as the first disturbance (marked by magenta lines). This second disturbance also causes reattachment. In the $D=10 \%$ case, the jets turn off only 0.1 cycle after they turn on, so the on and off disturbances act essentially as a single event. With $D=50 \%$ there is more time between the on and off disturbances, so they act as two separate events. The result is two effective disturbances per cycle, thereby doubling the disturbance frequency above the pulsing frequency and providing better separation control.

Fig. $5 c$ shows wavelet spectra for the $F=0.56, D=10 \%$ case. The convection velocity of the disturbances is about the same as with
$F=0.28$, but the period, $T$, is shorter, so the disturbances occupy a larger fraction of the cycle, and there is less time between disturbances for the separation bubble to grow. The time averaged profiles for the $F=0.28, D=50 \%$ and $F=0.56, D=10 \%$ cases in Fig. 3 are very similar, since these two cases have the same effective disturbance frequency (due to the doubling of the pulsing frequency with $D=50 \%$ discussed above). The frequencies of the largest peaks in Fig. $5 c$ are about $f L_{S} / U_{e}=2.6$, which is roughly double that in the $F=0.28$ cases, probably due to the higher pulsing frequency. As in the $F=0.28$ case, peaks associated with shear layer transition at $f L_{S} / U_{e} \approx 7$ are visible at Stations 1 and 2 . The wavelet spectra for the $F=1.12, D=10 \%$ case (not shown) are very similar to those with $F=0.56$, although the peak frequency is increased to about $f L_{S} / U_{e}=3.7$ since the pulsing frequency is higher. Upstream peaks at $f L_{S} / U_{e} \approx 7$ are still visible. Since the disturbance occupies nearly the entire cycle by the downstream stations with $F=0.56$, increasing to $F=1.12$ has little additional effect, as shown by the mean profiles in Fig. 3.

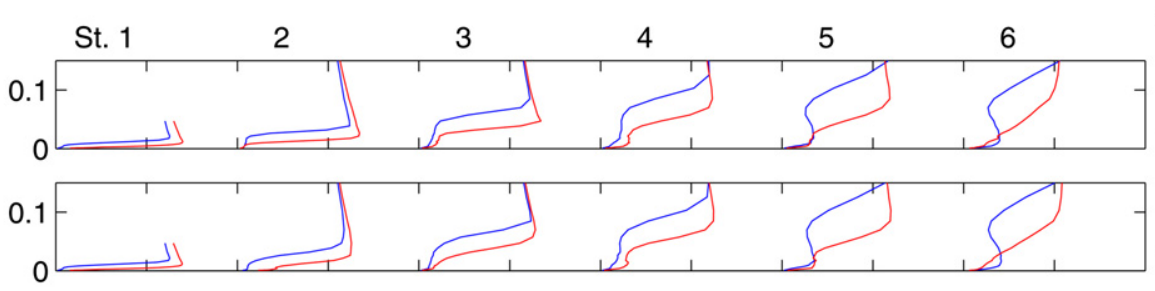

$t / T$

0.083

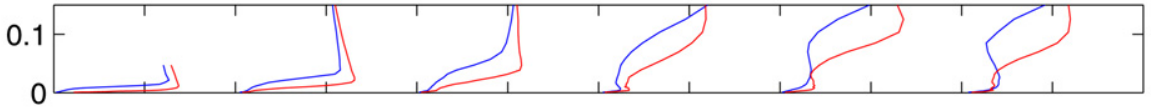

0.167
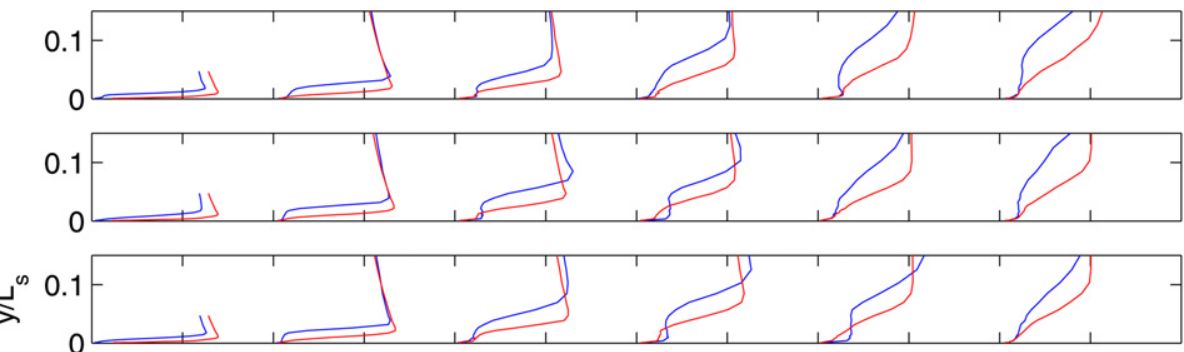

$$
\stackrel{\infty}{\lambda} 0 .
$$
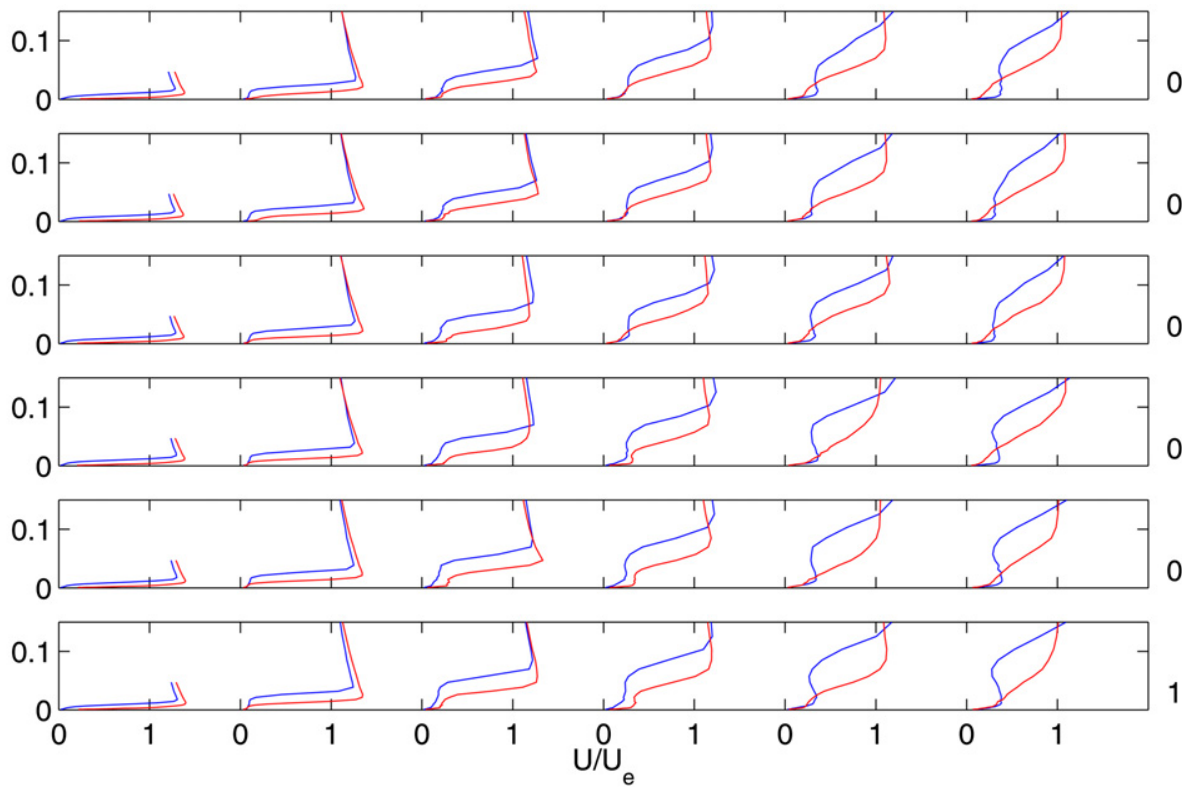

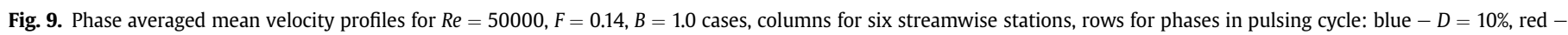
$D=50 \%$. (For interpretation of the references to colour in this figure legend, the reader is referred to the web version of this article.) 


\section{2. $R e=50000$}

Pressure profiles for the cases with $R e=50000$ are shown in Fig. 6. Without flow control the boundary layer does not reattach. As shown in Volino et al. [17], steady jets become effective when $B \geq 1.5$, and by $B=2$ the separation is nearly eliminated and the loss peak magnitude and width are greatly reduced. The integrated loss is about the same as in the no-jet case due to the loss associated with the pressure drop from the plenum to the jet exit. Flow turning is increased $12^{\circ}$ compared to the no-jet case. With pulsed jets and $B=1.0$, pulsing with $F=0.14$ has only a limited affect with $D=10 \%$, but with $F=0.14$ and $D=50 \%$ the boundary layer reattaches after a large separation bubble. Losses drop to about half the value in the no-jet case and flow turning increases $12^{\circ}$ from the nojet case. With $F=0.28$ and $D=10 \%$, the $C p$ profile is about the same as in the $F=0.14, D=50 \%$ case, but the loss peak is somewhat wider and the shift in the peak indicates about $3^{\circ}$ less flow turning. With $F=0.56$, Cp shows a slightly smaller separation bubble than at the lower frequencies, the losses are reduced to $40 \%$ of the no-jet case, and flow turning is $13^{\circ}$ higher than the no-jet case. Increasing the duty cycle from $10 \%$ to $50 \%$ has little effect when $F=0.56$. The losses with $F=0.56$ are about 2.4 times those in the high $R e$ reference case and there is about $3^{\circ}$ less flow turning due to the presence of the separation bubble and thicker boundary layer at $R e=50000$.

Fig. 7 shows time averaged velocity profiles for the steady and $D=10 \%, R e=50000$ cases. A large separation bubble without reattachment is present without flow control. Steady jets with $B=2.0$ eliminate the separation, in agreement with the $C p$ profiles of Fig. 6 . With $F=0.14$ the separation is nearly as large as in the no-jet case, but the shear layer is somewhat thicker and $u^{\prime}$ levels are higher. With $F=0.28$ there is a small separation bubble at Stations 3 and 4 , but the boundary layer reattaches downstream. With $F=0.56$ the boundary layer appears attached at all stations. Fig. 8 shows the effect of duty cycle. With $F=0.14$, increasing the duty cycle from $10 \%$ to $50 \%$ greatly reduces the separation bubble thickness, although a small bubble is still present when $D=50 \%$. When $F=0.56$, duty cycle appears to have no effect, in agreement with the pressure results of Fig. 6. The boundary layer is attached with both $D=10 \%$ and $50 \%$.

Phase averaged mean velocity is shown for the $F=0.14$ cases in Fig. 9. With $D=10 \%$, the boundary layer appears to be close to reattaching at $t / T=0.25,0.333,0.417$ and 0.5 at Stations 3 through 6 respectively. With $D=50 \%$, reattachment occurs at about the same phases as in the $D=10 \%$ case. The boundary layer then starts to reseparate, but at Stations 5 and 6 it reattaches again about half a cycle later. This second reattachment means less time for the separation bubble to grow, resulting in a thinner separation at all times. The first reattachment corresponds to the arrival of the disturbance created when the VGJs are turned on. The second reattachment corresponds to the disturbance created when the VGJs turn off. As in the $R e=25000$ case, increasing the duty cycle to $50 \%$ separates in time the disturbances created when the VGJs turn on and off, thereby doubling the frequency of separation control events above the pulsing frequency. The result, as shown in Fig. 9, is a reattached boundary layer for most of the cycle by the downstream stations. The same effect can be achieved by doubling the pulsing frequency to $F=0.28$ with $D=10 \%$.

\section{Conclusions}

The effect of vortex generator jets on the flow over the very high lift L1A airfoil was studied under low freestream turbulence conditions. Reynolds numbers based on suction surface length and nominal exit velocity of 25000 and 50000 were considered. Without flow control, the boundary layer separated and did not reattach. Flow control with VGJs was possible even at $R e=25000$. In agreement with previous studies, pulsed jets were more effective than steady jets. Effective separation control resulted in a $20 \%$ increase in lift and up to a $60 \%$ reduction in total pressure loss compared to baseline cases at the same Reynolds number. Loss values still remain higher than in high Reynolds number cases. Phase averaged velocity profiles and wavelet spectra show the boundary layer intermittently reattaching as disturbances pass and then separating between disturbances. Increasing the pulsing frequency reduces the time available for separation. When the time available is sufficiently small, the boundary layer remains attached at all times. At $R e=25000$, separation was nearly fully controlled for the full pulsing cycle when $F=0.5$ and $D=10 \%$. Higher frequency pulsing provided little additional benefit. At $R e=50000$ the separation bubble grows more slowly, so $F=0.3$ is sufficient. At lower pulsing frequencies, increasing the duty cycle to $50 \%$ is helpful. The most effective disturbances for controlling separation are created when the VGJs turn on and off, and increasing the duty cycle to $50 \%$ separates in time the on and off events, thereby doubling the frequency of flow control events above the pulsing frequency.

\section{Acknowledgements}

This work was sponsored by the National Aeronautics and Space Administration under grant NNC07IA10I. The grant monitors were Drs. Anthony Strazisar and James Heidmann of the NASA Glenn Research Center. The support of the United States Naval Academy Technical Support Department Shop and Fluids Laboratory is greatly appreciated.

\section{Nomenclature}

B

blowing ratio

Cp $\quad 2\left(P_{T}-P\right) / \rho U_{e}^{2}$, pressure coefficient

$C_{X} \quad$ axial chord

D duty cycle

F $\quad \mathrm{fL}_{\mathrm{j} \text {-te }} / \mathrm{U}_{\text {ave }}$

$f \quad$ frequency

$\mathrm{L}_{\mathrm{j} \text {-te }} \quad$ distance from VGJs to trailing edge

$\mathrm{L}_{\mathrm{S}} \quad$ suction surface length

$\mathrm{L}_{\varphi} \quad$ blade spacing (pitch)

P pressure

$P_{S} \quad$ upstream static pressure

$\mathrm{P}_{\mathrm{T}} \quad$ upstream stagnation pressure

$\mathrm{P}_{\mathrm{Te}} \quad$ downstream stagnation pressure

Re $\quad \mathrm{U}_{\mathrm{e}} \mathrm{L}_{\mathrm{s}} / v$, exit Reynolds number

$s \quad$ streamwise coordinate

$\mathrm{T} \quad$ period of jet pulsing cycle

$t$ time

$\mathrm{U}_{\mathrm{ave}} \quad$ average freestream velocity between VGJ holes and trailing edge

$\mathrm{U}_{\mathrm{e}} \quad$ nominal exit freestream velocity

$u^{\prime} \quad$ rms fluctuating streamwise velocity

$\mathrm{x} \quad$ axial distance from leading edge

$\varphi \quad$ coordinate along blade spacing

$\nu \quad$ kinematic viscosity

$\rho \quad$ density

$\psi \quad\left(P_{T^{-}}-P_{T e}\right) /\left(P_{T}-P_{S}\right)$, total pressure loss

\section{References}

[1] D.C. Wisler, The technical and economic relevance of understanding boundary layer transition in gas turbine engines NASA/CP-1998-206958. in: J.E. LaGraff, D.E. Ashpis (Eds.), Minnowbrook II, 1997 Workshop on Boundary Layer Transition in Turbomachines (1998), pp. 53-64.

[2] R.E. Mayle, The Role of laminar-turbulent transition in gas turbine engines, ASME J. Turbomachinery 113 (1991) 509-537. 
[3] R.J. Volino, Separated flow measurements on a highly loaded low-pressure turbine airfoil, ASME J. Turbomachinery 132 (2010) 011007.

[4] R.J. Volino, Separated flow transition under simulated low-pressure turbine airfoil conditions: Part 1 - Mean flow and turbulence statistics, ASME J. Turbomachinery 124 (2002) 645-655.

[5] R.J. Volino, Separated flow transition under simulated low-pressure turbine airfoil conditions: Part 2 -Turbulence spectra, ASME J. Turbomachinery 124 (2002) 656-664.

[6] T.J. Praisner, J.P. Clark, Predicting transition in turbomachinery - Part 1: a review and new model development, ASME J. Turbomachinery 129 (2007) 1-13.

[7] X.F. Zhang, M. Vera, H. Hodson, N. Harvey, Separation and transition control on an aft-loaded ultra-High-Lift LP turbine blade at low Reynolds numbers: low-speed Investigation, ASME J. Turbomachinery 128 (2007) 517-527.

[8] D.G. Bohl, R.J. Volino, Experiments with three-dimensional passive flow control devices on low-pressure turbine airfoils, ASME J. Turbomachinery 128 (2006) 251-260.

[9] J. Huang, T. Corke, F. Thomas, Plasma actuators for separation control on low pressure turbine blades, AIAA Paper 2003-1027 (2003)

[10] J.P. Johnston, M. Nishi, Vortex generator jets. Means for flow separation control, AIAA J. 28 (1990) 989-994.

[11] J.P. Bons, R. Sondergaard, R.B. Rivir, The fluid dynamics of LPT blade separation control using pulsed jets, ASME J. Turbomachinery 124 (2002) 77-85.

[12] R.J. Volino, Separation control on low-pressure turbine airfoils using Synthetic vortex generator jets, ASME J. Turbomachinery 125 (2003) 765-777.

[13] J.P. Gostelow, G.J. Walker, W.J. Solomon, G. Hong, N. Melwani, Investigation of the calmed region behind a turbulent spot, ASME J. Turbomachinery 119 (1997) 802-809.
[14] V. Schulte, H.P. Hodson, Prediction of the becalmed region for LP turbine profile design, ASME J. Turbomachinery 120 (1998) 839-846.

[15] J.P. Clark, Private Communication. Air Force Research Laboratory, 2007.

[16] J.P. Bons, J. Pluim, K. Gompertz, M. Bloxham, J.P. Clark, The application of flow control to an aft-loaded low pressure turbine cascade with unsteady wakes, ASME Paper GT2008-50864 (2008)

[17] R.J. Volino, O. Kartuzova, M.B. Ibrahim, Experimental and computational investigations of low-pressure turbine separation control using vortex generator jets, ASME Paper GT2009-59983 (2009).

[18] R.J. Volino, O. Kartuzova, M. Ibrahim, Experimental and computational investigations of separation and transition on a highly loaded low pressure turbine airfoil: Part 2 - High freestream turbulence intensity, ASME Paper IMECE2008-68776 (2008).

[19] R.J. Volino, O. Kartuzova, M.B. Ibrahim, Separation control on a very high lift low pressure turbine airfoil using pulsed vortex generator jets, ASME J Turbomachinery 133 (2011) 041021.

[20] J.A.B. Wills, The correction of hot-wire readings for proximity to a solid boundary, J. Fluid Mech. 12 (1962) 65-92.

[21] R.J. Volino, An investigation of the scales in transitional boundary layers under high freestream turbulence conditions, Exp. Fluids 38 (2005) 516-633.

[22] R.D. Stieger, H.P. Hodson, The transition mechanism of highly loaded lowpressure turbine blades, ASME J. Turbomachinery 126 (2004) 536-543.

[23] X.F. Zhang, H.P. Hodson, Effects of Reynolds number and freestream turbulence intensity on the unsteady boundary layer development on an ultra-high-lift low pressure turbine airfoil, ASME J. Turbomachinery 132 (2010) 011016 